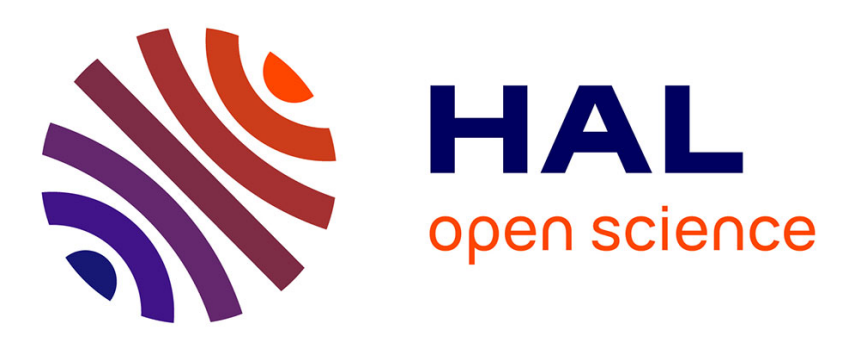

\title{
Towards a rapidly rotating liquid sodium dynamo experiment
}

Philippe Cardin, Daniel Brito, Dominique Jault, Henri-Claude Nataf, Jean-Paul Masson

\section{- To cite this version:}

Philippe Cardin, Daniel Brito, Dominique Jault, Henri-Claude Nataf, Jean-Paul Masson. Towards a rapidly rotating liquid sodium dynamo experiment. Magnetohydrodynamics c/c of Magnitnaia Gidrodinamika, 2002, 38, pp.177-189. hal-00000875

\section{HAL Id: hal-00000875 https://hal.science/hal-00000875}

Submitted on 25 Nov 2003

HAL is a multi-disciplinary open access archive for the deposit and dissemination of scientific research documents, whether they are published or not. The documents may come from teaching and research institutions in France or abroad, or from public or private research centers.
L'archive ouverte pluridisciplinaire HAL, est destinée au dépôt et à la diffusion de documents scientifiques de niveau recherche, publiés ou non, émanant des établissements d'enseignement et de recherche français ou étrangers, des laboratoires publics ou privés. 


\title{
Towards a rapidly rotating liquid sodium dynamo experiment
}

\author{
P. Cardin, D. Brito, D. Jault, H.-C. Nataf, J.-P. Masson \\ Laboratoire de Gophysique Interne et Tectonophysique, \\ Observatoire de Grenoble, Universit Joseph Fourier, \\ B.P. 53, 38041 Grenoble, France. \\ philippe.cardin@ujf-grenoble.fr
}

Juin 2002

\begin{abstract}
The main characteristics of the Earth's dynamo are reviewed. The combined actions of Coriolis and Lorentz forces lead to the so-called "magnetostrophic" regime. We derive an estimate of the power needed to sustain the magnetic field in this regime. We show that an experiment with liquid sodium can be designed to operate in the magnetostrophic regime. Such an experiment would bring most valuable informations on the mechanisms of planetary dynamos. In order to prepare this large-scale experiment and explore the magnetostrophic balance, a smaller scale liquid sodium set-up has been designed and is being built. It consists of a rapidly rotating spherical shell filled with liquid sodium, in which motions are set by spinning at a different rotation rate an inner core permeated by a strong magnetic field. We discuss the processes that can be explored with this new device.
\end{abstract}

\section{Introduction.}

\subsection{The magnetic fields in the solar system.}

Most planets of the solar system have or have had an internal magnetic field. Recent satellite missions have revealed that Mars probably had a magnetic field in its early history [1], while two moons of Jupiter, Io and Ganymede [2], show evidence for present magnetic activity. Explaining the origin and behaviour of planetary magnetic fields is a fascinating challenge. As a starting point, one may attempt to build a model of the geodynamo since the Earth's magnetic field is, by far, the best documented. 


\subsection{The Earth's magnetic field.}

The characteristics of the magnetic field of the Earth are known over a wide range of time scales. In historical times, observatory records permit to monitor the secular variation of magnetic structures, with typical drift velocities of 0.1 $\mathrm{mm} / \mathrm{s}$. Sudden variations of the internal magnetic field, affecting the entire core surface, have also been discovered in these records, the so-called "jerks", while the total intensity of the field displays large fluctuations and has dropped by more than $30 \%$ in the past 2000 years, as recorded in archaeological artefacts. The most striking property of the Earth's magnetic field, as seen at present, but also in the paleomagnetic records over several hundred of million years, is that it is dominated by a dipole component, whose axis is aligned with the axis of rotation of the Earth. Just as fascinating is the evidence that this dipole has changed polarity many times over the Earth's history, the last inversion having taken place only 780,000 years ago [3]. Models of the geodynamo aim at explaining this rich set of behaviours, starting with the dominant dipole character of the field.

\subsection{The mechanism.}

Since the pioneer work of Larmor, Bullard and Elsasser, it is widely accepted that the Earth's magnetic field is created by self-induction in its core. In an electrically conducting liquid, fluid motions in a magnetic field produce electrical currents, which in turn feed the magnetic field. When the induction of the magnetic field overcomes its diffusion (i.e., for a large enough magnetic Reynolds number), a self-sustained magnetic field is obtained. The main component of the $3480 \mathrm{~km}$-radius core is iron. With temperatures of the order of 5000 $\mathrm{K}$, most of the core is liquid, but under the effect of pressure, it crystallizes and the $1220 \mathrm{~km}$-radius inner core is solid, as revealed by seismology. Material properties of the core are deduced from seismological observations, high pressure experiments, $a b$ initio calculations, and thermodynamic theory. It turns out that its electrical conductivity, thermal conductivity, and viscosity are not very different from those of liquid iron at atmospheric pressure [4]. In particular, the magnetic diffusivity $\eta$ is about $1 \mathrm{~m}^{2} / \mathrm{s}$. With typical velocities $U$ of $0.1 \mathrm{~mm} / \mathrm{s}$, one estimates a magnetic Reynolds number $R_{m}=U a / \eta \sim 300$, where $a$ is the core radius, suggesting that dynamo action can indeed take place in the core and be responsible for the Earth's magnetic field.

\subsection{The energy source.}

Convection is the dominant mechanism through which the Earth cools. As the temperature of the core decreases (on the time scale of billions of years), iron crystallizes at the surface of the solid inner core. Latent heat is released and contributes to the energy source. The light elements that enter the composition

of the core are left (in part) in the liquid phase and cause convective motions through compositional buoyancy. Both latent heat released by inner core so- 
lidification and gravitational heat due to inner core differentiation currently contribute to the dynamo power [5]. However, the crystallization of the inner core might have started less than 2 billion years ago [6]. Paleomagnetic records indicate that a magnetic field was already present on Earth more than 3 billion years ago. Hence, thermal convection (due to secular cooling) alone is probably able to power the dynamo. Less than $10^{11} \mathrm{~W}$ is needed to maintain the Earth's magnetic field, while heat flowing out of the core is probably in excess of $4 \cdot 10^{12}$ W. Nevertheless, other energy sources may contribute. Though precession and tides (under the gravitational influence of the Moon and Sun) act on the fluid core at diurnal periods, both phenomena may also, through nonlinear interactions, induce motions that have long time scales and participate in the dynamo mechanism.

\subsection{Some characteristics of the core.}

Whatever the energy source, it is clear that motions in the core are strongly influenced by the rotation of the Earth. Indeed, the viscosity in the liquid core is of the order of $10^{-6} \mathrm{~m}^{2} / \mathrm{s}$, similar to that of water. The Ekman number $E$, which compares the viscous forces to the Coriolis force (see the definition at the end of section 1.1 ), is as small as $10^{-15}$. With such a low Ekman number, convective motions are submitted to the Taylor-Proudman constraint and take the form of narrow columns, with their axis aligned with the axis of rotation. At the onset of convection, the typical diameter of the columns is of the order of $a E^{1 / 3}$ as convection has to call upon viscous effects in the fluid to alleviate the Taylor-Proudman constraint and enable radial heat transport. Fully developed convection retains this columnar signature, but wider columns can form as an inertial regime sets in. A turbulent inverse cascade develops and feeds a sizeable zonal flow [7]. Viscous dissipation is several orders of magnitude smaller than Joule dissipation. Then, the Lorentz force and Coriolis force tend to be of the same order, leading to the so-called magnetostrophic regime (see review [8]), where geostrophic motions easily arise. Thus, zonal motions around the rotation axis are thought to play a particular role.

\subsection{Numerical models of the geodynamo.}

The past 10 years have seen the rise of fully dynamical numerical models of the dynamo. In these 3D models, both the equations of motions and those of magnetic induction are solved. In order to be tractable, the models assume a relatively small rotation rate, i.e. an Ekman number of the order of $10^{-4}$. Wide convective columns are produced and the Taylor-Proudman constraint is not very strong. Surprisingly, the columns produce rather naturally a magnetic field with a dominant dipolar component (but see [9]). Furthermore, the intensity of the magnetic field scaled with the rotation rate is comparable to that of the Earth [10]. This could be the indication of some universal behaviour. However, the range of parameters that can be accessed in numerical models remains very far from that of the core. In particular, while the magnetic diffusivity is $10^{6}$ 
larger than viscosity in the core, it is never more than 10 times larger in the models, which means that the magnetic field and the velocity field operate on the same length scale. Furthermore, viscous dissipation remains the dominant dissipative process, in contrast to what is expected for the core. Attempts to remove viscosity altogether and jump onto a Joule dissipation-controlled magnetostrophic dynamo branch have not been successful yet, while efforts to mimic subgrid turbulent processes are hampered by our lack of knowledge about the organization of turbulence under the combined effects of rotation and magnetic field.

\subsection{The need for experiments.}

Our vast ignorance of the magnetohydrodynamics of a liquid that has the properties of the Earth's core leaves room for laboratory exploration, especially since dynamo experiments use liquid metal, whose properties (viscosity and magnetic diffusivity) fall into the proper range. Like for all other dynamo experiments [11], the first challenge is to reach a large enough magnetic Reynolds number. A large tank and a large driving power are required. The second challenge is to impose a global rotation to the tank, since the Coriolis force plays a key role in the geodynamo. In order to further mimic the basic equilibrium of the core, we want the Lorentz force to balance the Coriolis force, which means that the magnetic field is rather large, implying an even larger driving power than in previous experiments.

\subsection{What forcing?}

Pursuing further the analogy with the core, one is tempted to rely on thermal convection to produce the motions in the tank. However, convection is an inefficient energy converter. Extrapolations of convective velocities measured in small-scale experiments using liquid gallium [7] indicate that for a given size and power, convective velocities would be two orders of magnitude smaller than motions generated with direct mechanical forcing. It is therefore more reasonable to produce motions in the tank by injecting kinetic energy directly. At first order, the nature of the forcing is irrelevant since the dominant force balance is between the Coriolis and Lorentz forces. Differential rotation of an inner object can provide the required forcing.

The aim of the present article -following up a previous discussion along the same lines [12]- is to show that one can design an experiment that captures the essential ingredients of the geodynamo. In the next section, we define the dimensionless numbers that allow to compare the magnetic and the rotation forces both in the Earth's core and in a laboratory experiment at different time scales. The analysis provides an estimate of the size and power requirements of such an experiment. Then, we describe a smaller-scale experiment, which we have designed to further document the competition between the constraints given by the rotation and an ambient magnetic field. We conclude with a discussion about the goal of experimental endeavours in the geomagnetic context. 


\section{Investigating the competing roles of the rota- tion and magnetic forces.}

We sum up the influence of the rotation and magnetic forces on the dynamics of a fluid enclosed in a rapidly rotating tank and permeated by a magnetic field. We first focus our analysis on the large scale part of the motions. We assume that the cavity has spherical boundaries even if most of our remarks have more general scope. The characteristic length scale is then given by the outer radius $a$. We investigate different timescales beginning with the shortest ones in a body of planetary size.

\subsection{Diffusionless waves and the Taylor's constraint.}

We first filter out seismic waves and assume incompressibility. Earth's tides and precession and nutation of the Earth's rotation axis generate motions with diurnal period inside the Earth's core. They obey the equation for inertial waves:

$$
\rho\left(\frac{\partial \mathbf{u}}{\partial t}+2 \boldsymbol{\Omega} \times \mathbf{u}\right)=-\nabla p,
$$

where $\mathbf{u}$ is the velocity, $p$ the pressure, $\rho$ the density and $\boldsymbol{\Omega}$ the angular velocity.

Now, if the Earth were not rotating, Alfvèn waves would propagate inside the core. Denoting by $\mathbf{B}$ and $\mathbf{b}$ respectively the ambient and induced magnetic field and by $\mathbf{j}$ the electrical current density, we obtain the following set of equations,

$$
\begin{gathered}
\rho \frac{\partial \mathbf{u}}{\partial t}=-\nabla p+\mathbf{j} \times \mathbf{B}, \\
\frac{\partial \mathbf{b}}{\partial t}=\nabla \times(\mathbf{u} \times \mathbf{B}), \nabla \times \mathbf{b}=\mu_{0} \mathbf{j},
\end{gathered}
$$

which are obeyed by Alfvèn waves with the frequency

$$
\omega_{a}=\frac{B}{\left(\mu_{0} \rho\right)^{1 / 2} a},
$$

where $\mu_{0}$ is the magnetic permeability of vacuum.

Comparing the period of the inertial waves and of the Alfvèn waves gives a first measure of the relative strengths of the constraints imparted to the fluid by the rotation and magnetic forces

$$
\lambda=\frac{\omega_{a}}{\Omega}=\frac{B}{\Omega\left(\mu_{0} \rho\right)^{1 / 2} a} .
$$

The importance of the parameter $\lambda$ is demonstrated by an analysis $[13$, pp 233-236] of the diffusionless waves propagating when the fluid is both in rigid rotation and permeated by an uniform magnetic field. If $\lambda \gg 1$, Alfvèn waves are only slightly modified by the Coriolis force. If instead $\lambda \ll 1$, a new family 
of slow "magnetostrophic" waves for which inertia is negligible is exhibited in addition to the inertial waves. Inside the Earth's core, $\lambda \ll 1$ (see below) and the inertial waves are probably not strongly coupled to the core dynamics responsible for magnetic field generation. Then inertia is important only for the geostrophic motions that obey the following balance:

$$
2 \rho\left(\boldsymbol{\Omega} \times \mathbf{u}_{g}\right)=-\nabla p .
$$

Geostrophic motions $\mathbf{u}_{g}$ accelerated by the magnetic force arise indeed as Taylor's condition is violated. The latter constraint,

$$
\forall \mathbf{u}_{g}, \quad \int_{V_{\Gamma}} \mathbf{u}_{g} \cdot(\mathbf{j} \times \mathbf{B}) d V=0,
$$

(where $V_{\Gamma}$ denotes the fluid volume where geostrophic contours are extant) gives the solvability condition for the equation of magnetostrophic equilibrium:

$$
2 \rho(\boldsymbol{\Omega} \times \mathbf{u})=-\nabla p+\mathbf{j} \times \mathbf{B} .
$$

These geostrophic motions $\mathbf{u}_{g}$ are special Alfvèn waves and are usually referred to as torsional Alfvèn waves. Arguably, these waves are indirectly observed at the core surface and are responsible for time changes in the Earth's magnetic field [14]. Their period (of the order of 50 years) yields an estimate of the intensity of the magnetic field $B_{s}$ threading the geostrophic cylinders in the core interior (see (4) with $B$ replaced by $B_{s}$ ), which turns out to be close to the magnetic field intensity at the core surface $\left.B\right|_{r=a}=5 \cdot 10^{-4} \mathrm{~T}$.

Investigating torsional Alfvèn waves with numerical models of the geodynamo is rather difficult. In these models, the magnetic Prandtl number $P_{m}=$ $\nu / \eta$, where $\nu$ is the kinematic viscosity, is chosen quite high to enable dynamo action. Then, viscous breaking can be so efficient as to prevent the propagation of the waves. Unfortunately, experimental investigation of the magnetostrophic regime (and thus of the Alfvèn torsional waves) is challenging also because it requires that $\lambda \ll 1$. Furthermore, it is difficult to devise experiments where the magnetic diffusion time $\tau_{J}$ (roughly estimated here as $a^{2} / \eta$ ) is not much shorter than the period $T_{a}$ of the waves:

$$
\frac{T_{a}}{\tau_{J}}=\frac{\eta\left(\mu_{0} \rho\right)^{1 / 2}}{a B} \ll 1 .
$$

The condition $\lambda \ll 1$ defines a magnetostrophic regime. Whereas the value of the Ekman number $E\left(E=\nu / \Omega a^{2}\right)$ is very significant in numerical models, as already noted above in the discussion about torsional waves, the condition $\lambda \ll 1$ indicates more specifically than the expression $E \ll 1$ what we mean by rapid rotation in the experimental dynamo context.

In a dynamo experiment, the field is not imposed. Therefore, we need a way to evaluate its equilibrium intensity. This is the purpose of the next section. 


\subsection{The Elsasser numbers.}

On long timescales, diffusion cannot be neglected. A second dimensionless number comparing the rotation and magnetic forces (see (8)) can be derived. The Elsasser number is generically defined as:

$$
\Lambda=\frac{j B}{2 \rho \Omega U},
$$

where an estimate of the current density $j$ remains to be specified. If the main magnetic field is imposed, the typical intensity of the electrical currents is best estimated from the Ohm's law:

$$
\mathbf{j}=\sigma(\mathbf{E}+\mathbf{u} \times \mathbf{B}) .
$$

We thus obtain a first Elsasser number:

$$
\Lambda_{i}=\frac{\sigma B^{2}}{2 \rho \Omega}=\lambda^{2} \frac{\Omega a^{2}}{2 \eta} .
$$

In a self-consistent geodynamo model, the magnetic field is induced by electrical currents circulating within the core. Let us note:

$$
\gamma^{2}=\frac{\eta}{a^{2}} \frac{\int_{V} \frac{j^{2}}{\sigma} d \tau}{\int_{\infty} \frac{B^{2}}{\mu_{0}} d \tau} .
$$

The dimensionless factor $\gamma$ is well known in the dipolar case, $\gamma=\pi$. We relied on kinematic dynamo calculations to estimate it in more realistic cases (the Gubbins and Pekeris dynamo models), but still with smooth velocity fields. We find $\gamma \simeq 6$ near the onset of dynamo action and $\gamma \simeq R_{m}^{1 / 2}$ in the limit of high magnetic Reynolds number. Gubbins et al. [15] obtained larger values at the onset of dynamo action $(\gamma \simeq 15--30)$ while studying dynamo models where the toroidal zonal part is dominant. A second Elsasser number is given by

$$
\Lambda_{d}=\frac{\gamma B^{2}}{2 \mu_{0} a \rho \Omega U}=\frac{\sigma \gamma B^{2}}{2 \rho \Omega R_{m}}=\frac{\gamma}{R_{m}} \Lambda_{i} .
$$

Both $\Lambda_{i}$ and $\Lambda_{d}$ take diffusion into account. $\Lambda_{i}$ is the appropriate number to discuss models with imposed magnetic field whereas $\Lambda_{d}$ is more adequate to dynamo studies. In a first approximation, it is often assumed that the Elsasser number $\Lambda_{d}$ is of order unity inside the Earth's core (see the Introduction).

This assumption yields a second estimate for the intensity of the magnetic field in the core interior:

$$
\left.B \simeq 5 \cdot 10^{-3} T \simeq 10 B\right|_{r=a}
$$

(with $\gamma=20, R_{m}=300$ ). It is not incompatible with the former estimate, if the main part of the magnetic field is parallel to the geostrophic contours within 
the core. From (15), we can now estimate the parameter $\lambda$ inside the Earth's core as $\lambda \simeq 2 \cdot 10^{-4}$.

Finally, the characteristics of the geodynamo that we find important to reproduce in a laboratory experiment are $\Lambda_{d}=1$ and $\lambda \ll 1$. They define a magnetostrophic regime. The parameter $\Lambda_{d} / \lambda^{2}$ gives the rotation rate whereas $\Lambda_{d} / \lambda$ provides a measure of the magnetic field intensity, which is independent of the rotation rate. Thus, the power requirements scale with $\left(\Lambda_{d} / \lambda\right)^{2}$.

\subsection{Saturation of the magnetic field intensity.}

An estimate of the ohmic heating is given by

$$
P_{J}=\frac{4}{3} \pi a^{3} \frac{B^{2}}{2 \mu \tau_{J}},
$$

where the Joule dissipation time $\tau_{J}$ is accurately estimated now as $a^{2} / \gamma^{2} \eta$. From (5) and (14), we obtain:

$$
P_{J}=\frac{8 \pi \rho \eta^{3}}{3 a} R_{m}^{2}\left(\frac{\Lambda_{d}}{\lambda}\right)^{2} .
$$

Let us apply (17) to the geodynamo case in order to check that it gives a reasonable estimate of the power dissipated by geophysically relevant dynamos. With $R_{m}=300, \Lambda_{d}=1$ and $\lambda=2 \cdot 10^{-4}$, we find $P_{J}=5 \cdot 10^{10} \mathrm{~W}$. It is probably more realistic that the lower bound derived from models of the magnetic field at the core surface, which only comes up to $P_{J} \geq 15 \cdot 10^{8} \mathrm{~W}[16]$.

Keeping $\Lambda_{d}$ and $\lambda$ constant, the dissipated power $P_{J}$ scales as $R_{m}^{2}$ in contrast to the hydrodynamical case where it scales as $R_{m}^{3}$. From (14), we note that $\Lambda_{i}$, and thus the magnetic field intensity, increase with $R_{m}$ if $\Lambda_{d}$ is to remain of order unity in a rotating dynamo experiment. From kinematic modelling, it has been suggested that the geometry of the magnetic field also changes with $R_{m}$, the lines of force of the field being more and more aligned with the streamlines as $R_{m}$ increases. We discuss now a flow geometry reproducible in the laboratory, which is associated with a well understood dynamo mechanism.

\subsection{A kinematic approach of rotation dominated regimes.}

Braginsky investigated models where the zonal circulation (flow along circular contours) is dominant supposedly as the result of rotation forces, which have no other role in this kinematic approach. Zonal motions appear indeed ubiquitous in rotating flow experiments. Our rotating convection experiment with gallium gives us an example where large scale zonal motions with velocities comparable to the convective velocities arise as the result of an inverse cascade [7]. The Braginsky model explains how a dipolar magnetic field symmetrical about the rotation axis may emerge [17, chap.8]. Then, Kumar and Roberts [18] devised a numerical model to illustrate the theory of Braginsky. There have been numerous studies of this model [15]. The difficulty to devise an experiment along 
these lines stems from the high value of the magnetic Reynolds number $R_{m c}$ at the onset of dynamo action. The challenge is to decrease the proportion of zonal motions to lower $R_{m c}$ whilst keeping these motions intense enough to make the Braginsky analysis valid. Originally, Kumar and Roberts chose the non-zonal velocity field so as to mimic the velocity field at the onset of convection. Because it appears to be very difficult to build a convective dynamo in a laboratory (see the Introduction), we are looking for another way to generate a velocity pattern with similar properties.

\subsection{A "magnetostrophic" dynamo experiment.}

We now have all the ingredients to propose an experimental dynamo that would operate in the same regime as the geodynamo. A spherical tank of radius $a$ is rotated with the angular velocity $\Omega$. An inner sphere of radius $b$ rotates at a different angular velocity $\Omega+\Delta \Omega$ but along the same axis. The expression (17) of the power dissipated by Joule effect yields a clear guide for dimensioning the experiment. The $\rho \eta^{3}$ term means that liquid sodium is the most suitable working liquid (at $\mathrm{T}=120{ }^{\circ} \mathrm{C}, \rho=920 \mathrm{~kg} \cdot \mathrm{m}^{3}$ and $\eta=0.08 \mathrm{~m}^{2} \mathrm{~s}^{-1}$ ). The $a$ term in the denominator implies that a smaller experiment will need a larger power, for a given $R_{m}$.

As a starting point, we choose $a=1 \mathrm{~m}$. Then, the ohmic heating (in watts) is estimated as

$$
P_{J} \simeq 4 R_{m}^{2}\left(\frac{\Lambda_{d}}{\lambda}\right)^{2} .
$$

Assuming $\Lambda_{d}=1$, we find that it is difficult to make $\lambda$ very small while keeping $R_{m}$ large enough and $P_{J}$ small enough. Choosing $\lambda=0.25$ and $R_{m}=100$ should enable us to obtain a dynamo experiment with magnetostrophic characteristics even if inertial waves would play a much more important role in the generation of the magnetic field than in the Earth's core. These parameters correspond to $P_{J}=640 \mathrm{~kW}$. Let us now show that these values of $\Lambda_{d}, \lambda$ and $R_{m}=100$ are realizable. Assuming $\gamma=6$, we find $\Omega=43 \mathrm{rad} . \mathrm{s}^{-1}$. The intensity of the magnetic field that should be produced is $B=0.36 \mathrm{~T}$. If $R_{m}=\Delta \Omega b a / \eta$, we obtain $R_{m}=100$ with $b=0.5 \mathrm{~m}$ and $\Delta \Omega=16 \mathrm{rad} . \mathrm{s}^{-1}$. The ohmic dissipation time $\tau_{J}(0.35 \mathrm{~s})$ would be only half the period of the Alfvèn waves. If the power injected in the tank is not removed, the temperature will increase at a rate of $0.1 \mathrm{~K} / \mathrm{s}$. However, because the tank rotates rapidly, heat can be removed rather efficiently at its surface.

From our analysis, such an experiment would contain the basic ingredients of planetary dynamos. We expect that a strong dipolar magnetic field will be produced. With adequate instrumentation, one could investigate the organization of motions in the tank, the link with magnetic features at its surface, the role of torsional Alfvèn waves, the occurrence of global and rapid events

alike geomagnetic jerks and magnetic field reversals, and the characteristics of turbulence, to quote a few. 
The size and power of the experiment we envision are only slightly larger than those of the successful dynamo experiments of Riga [19] and Karlsruhe [20]. While the threshold for dynamo action could be accurately predicted in these two cases, it is much more difficult in our experiment, because we target a regime in which the magnetic field strongly controls the flow itself. Thus, the mechanical forcing we suggest would only be efficient when a strong magnetic field is present. In order to refine our predictions, we need to test several assumptions that were used, in particular the neglect of viscous dissipation and the forcing efficiency in the strong magnetic field regime. We have designed a prototype that should help us answering these questions.

\section{The prototype DTS.}

DTS (standing for "Derviche Tourneur Sodium") is a small-scale experiment that displays most features of the magnetostrophic dynamo experiment we propose. It contains around 40 liters of liquid sodium. Consequently, we do not expect to reach the dynamo regime but we aim at discovering some features of magnetostrophic flows. The goals of this experiment are dual: first, DTS is a small prototype where we can test and try several options that would be implemented in a future, larger, dynamo experiment. Second, DTS is an experiment devoted to the study of MHD turbulence in the presence of a strong magnetic field and rotation in the magnetostrophic regime.

\subsection{Geometry and kinematics.}

As already mentioned in the Introduction, quasi-geostrophic turbulence observed in thermal convection experiments is influenced by the geometry of the rotating container. In order to be able to apply our results to planetary situations, we choose to study the flow in a spherical shell of aspect ratio $b / a=0.35$, where $b$ is the radius of the inner sphere.

The fluid is moved by differential rotation between the inner and outer boundaries. Both spherical boundaries are designed to rotate at rates between -2000 and 2000 rotations per minute (rpm). In contrast with the case when the outer boundary is at rest - the so-called spherical Couette flow, which has been studied experimentally and numerically (for example [21]) — the case with

a rotating outer boundary has received little attention and we are aware of only few experimental results [22]. We plan to study first a water version of DTS. Qualitatively, we expect that vortices parallel to the axis of rotation are generated by the shear between the two concentric spheres. Shear layer instabilities in the rotating case are shown in Fig. 1.

Another kind of fluid flow we plan to study with DTS is the response of the fluid to an abrupt change in rotation rate, following the ideas of the group in Perm [23]. The fluid response of a spin up/spin down has been studied extensively (see review [24]) but little is known about the turbulence associated with these regimes. 


\subsection{Imposed magnetic field.}

Because we are not expecting to observe a self-sustained magnetic field in DTS, only an imposed magnetic field may change the properties of the hydrodynamical flow of liquid sodium. In order to investigate the action of Lorentz forces on the rapidly rotating flow, we impose a quasi-dipolar magnetic field induced by a permanent magnet, which fills the inner core. Its intensity $(0.15 \mathrm{~T}$ at the north pole of the inner core) is strong enough to make the magnetic forces have an important influence on the fluid motion. It falls off rapidly, as $r^{-3}$, from the inner sphere to the outer boundary. Let us take the intensity $(22 \mathrm{mT})$ of the magnetic field on the axis and at the distance $b+(a-b) / 2$ of the centre as a typical value of $B$. Then, changing the rotation rate from $20 \mathrm{rpm}$ to 2000 rpm, we decrease $\lambda$ from 1.4 to $1.410^{-2}$ and $\Lambda_{i}$ from 1.1 to $1.110^{-2}$. We are therefore expecting that the fluid dynamics within the cavity will have some magnetostrophic characteristics. Once again, little is known about this regime. It is worth noting that the imposed magnetic field carried by the inner core will act as a magnetic propeller, the fluid flow following the magnetic field lines. To enhance this effect, we have chosen to enclose the permanent magnet in a casing made out of copper, whose electrical conductivity is three times that of liquid sodium.

\subsection{MHD numerical calculation of the axisymmetric flow.}

We have undertaken a numerical study of the axisymmetric geostrophic spherical Couette flow [25]. We have computed the flow generated by a slight differential rotation of an inner core carrying a dipolar magnetic field, in a rotating spherical shell. For $\Lambda_{i} \ll 1$, the axisymmetric flow consists of the geostrophic solution of Proudman, and of a Stewartson viscous layer which develops along the cylinder tangent to the inner core and parallel to the axis of rotation. For a large difference in the rotation rate, this layer should be destabilised as asserted in the previous section. This regime will be studied experimentally in DTS for a very large rotation rate $(2000 \mathrm{rpm})$. For larger Elsasser number, we discovered numerically that the liquid rotates faster than either spheres inside a ring attached to a closed magnetic field line, as shown in Fig. 2. Finally, when the outer sphere is at rest, we can study the magneto-viscous problem and check the asymptotic laws derived in [26]. The flow should lose its axisymmetry for a large enough differential rotation of the inner core, and we will study the instabilities that then appear.

\subsection{Experimental set-up.}

The experimental set-up, shown in Fig. 3, has been designed to be installed in a room dedicated to sodium experiments. Sodium is kept solid or liquid under argon atmosphere in a gas-tight reservoir at the base of the set-up. Before every experimental run, the sodium loop is heated up and sodium is driven up by argon overpressure towards the spherical container through stainless steel 
tubes. At the end of the fill-up operation, the automatic electromagnetic valve is closed and the liquid sodium is therefore kept in the upper part of the set-up, namely the spherical shell.

The rotating spherical container is composed of a stainless steel outer sphere of radius $0.21 \mathrm{~m}$ and thickness $0.005 \mathrm{~m}$, and an inner copper sphere of outer radius $0.074 \mathrm{~m}$ and thickness $0.01 \mathrm{~m}$. The liquid sodium is therefore enclosed between a quasi-insulating outer boundary (stainless steel is ten times less conducting than sodium) and an inner conductive boundary as seen in section 2.2. The inner sphere is filled with permanent magnets, which impose a strong, mainly dipolar, magnetic field on the fluid flow.

Brushless motors 1 and 2 (see Fig. 3), $10 \mathrm{~kW}$ each, make the inner core and the outer shell rotate at different rates. The differential motion is transmitted by a shaft connected to a magnetic coupling, which is used to transfer the mechanical power from motor 2 to the inner core through the stainless steel wall of the sodium container. Twelve pairs of permanent magnets are mounted on both sides of the wall and rotation of the outer ones drives the ones attached to the shaft in liquid sodium. The magnetic coupling is designed to transfer a maximum mechanical torque of $75 \mathrm{Nm}$.

The spherical shell is located in a thermostated box that keeps the temperature constant at $120^{\circ} \mathrm{C}$. During an experiment, air is pumped into the box to cool down the spherical shell. Outside the chamber, a set of 36 electrical slip rings enables us to perform measurements in the rotating frame during the experiments.

The temperature at various points in the sodium loop, as well as pressure and sodium level will be monitored. In case of emergency, the automatic electromagnetic valve will open and liquid sodium will fall down into the reservoir.

\subsection{Instrumentation.}

We have recently shown that ultrasonic Doppler velocimetry is a reliable technique for measuring velocities in opaque liquids such as gallium [28]. Our preliminary tests in sodium are promising and we intend to implement this technique in DTS. A couple of ultrasonic probes will be installed on the rotating outer shell and will measure radial and azimutal velocities of the fluid as a function of radius, at various heights along the rotation axis.

Measuring the induced magnetic field will be a challenging task in our experiment, because of the large imposed magnetic field and moderate magnetic Reynolds number. We plan to install a magnetic probe in the fixed frame and measure the field continuously as the spherical shell rotates beneath it. Bundles of stronger induced magnetic fields are expected at the top of dynamical columnar structures aligned with the axis of rotation.

During experiments, the highly electrically conductive liquid sodium is in contact with the outer shell made of stainless steel, an electrical insulator as seen by the fluid. Several copper electrodes pierce the outer boundary, and their tip is only $1 \mathrm{~mm}$ away from liquid sodium. The differences in electrical 
potential between these electrodes will help constraining the electrical currents generated in the bulk of the fluid.

Local dynamical pressure and global torque measurements will be performed as well.

\section{Conclusions.}

After the exciting success of the experimental dynamos in Riga and Karlsruhe, it is felt that the priority should be the investigation of situations in which the magnetic field strongly affects the flow that creates it. The type of dynamo experiment we propose falls into this category. Its originality lies in the strong imposed global rotation. Rotation clearly plays a dominant role in planetary dynamos. The competition between Coriolis and Lorentz forces imparts peculiar characteristics to the flow. Furthermore, it is likely that rotation favors the apparition of a magnetic field. Numerical models of the Earth's dynamo provide a good illustration of this. However, these models cannot access the range of parameters that characterizes the geodynamo. Important phenomena such as torsional Alfvèn waves are excluded.

Experiments with liquid sodium permit the exploration of regimes that are characteristic of planetary dynamos. The "magnetostrophic" regime, in which the Coriolis and Lorentz force are in balance is of particular interest. This regime can be attained in a dynamo experiment, and we have derived an estimate of the power and ingredients needed for such an experiment.

The magnetostrophic balance can also be tested in smaller scale experiments with an imposed magnetic field. We have designed and are installing an experiment (DTS) with this goal in mind, hoping that it will provide new clues to the magnetohydrodynamics of planetary cores. DTS will also serve as a prototype, in which technological solutions, measurement methods, and various forcings can be tested.

\section{Acknowledgements.}

This work is supported by Ministre de l'Education Nationale, de la Recherche et de la Technologie (Fonds National de la Science) and by Centre National de la Recherche Scientifique. The design of the DTS experiment has been performed by SERAS (CNRS-Grenoble). We thank A. Alemany for his cooperation in the design of the DTS experiment, E. Dormy for the computations and images of Fig. 2, F. Busse for reading a preliminary version of the article and K.H. Rädler for his careful review of this paper.

\section{References}

[1] Stevenson D. J. Mars' core and magnetism. Nature, vol. 412 (2001), pp. 214-219. 
[2] Kivelson M. G., Khurana K. K., Russell C. T., Walker R. J., Warnecke J., Coroniti F. V., Polanskey C., Southwood D. J. And G. Schubert. Discovery of Ganymede's magnetic field by the Galileo spacecraft. Nature, vol. 384 (1996), pp. 537-541.

[3] Merrill R. T., McElhinny M. W. and P. L. McFadden. The Magnetic Field of the Earth. Academic Press, (San Diego, 1996).

[4] Poirier J. P. Introduction to the Physics of the Earth's Interior. Cambridge University Press, (Cambridge, 1991).

[5] Lister J. R. And B. A. Buffett. The strength and efficiency of thermal and compositional convection in the geodynamo. Physics of the Earth and Planetary Interiors, vol. 91 (1995), pp. 17-30.

[6] Labrosse J., Poirier J.-P. And J.-L. Le MouËL. The age of the inner core. Earth and Planetary Science Letters, vol. 190 (2001), pp. 111-123.

[7] Aubert J., Brito D., Nataf H.-C., Cardin P. and J.-P. Masson. A systematic experimental study of rapidly rotating spherical convection in water and liquid gallium. Physics of the Earth and Planetary Interiors, vol. 128 (2001), pp. 51-74.

[8] Fearn D. R. Hydromagnetic flow in planetary cores. Rep. Prog. Phys., vol. 61 (1998), pp. 175-235.

[9] Grote E., Busse F. H. And A. Tilgner. Convection-driven quadrupolar dynamos in rotating spherical shells. Physical Review E, vol. 60 (1999), pp. 5025-5028.

[10] Christensen U., Olson P. and G. A. Glatzmaier. Numerical modelling of the geodynamo: a systematic parameter study. Geophysical Journal International, vol. 138 (1999), pp. 393-409.

[11] Nataf H.-C. Dynamo and convection experiments In Earth's Core and Lower Mantle, edited by C. A. Jones, A. M. Soward and K. Zhang, Taylor and Francis, in press.

[12] Jault D., Cardin P. And H.-C. NataF . Geodynamo and MHD. In Transfer Phenomena in Magnetohydrodynamic and Electroconducting Flows, edited by A. Alemany, Ph. Marty and J.-P. Thibault, Kluwer academic publishers, (Dordrecht, 1999) pp. 17-30.

[13] Fearn D.R., Roberts P. H. And A. M. Soward. Convection, stability and the dynamo. In Energy Stability and Convection, edited by G. P. Galdi and B. Straughan, Longman scientific and technical, (Harlow, 1988) pp. 60-324.

[14] JAULT D. Electromagnetic and topographic coupling, and LOD variations. In Earth's Core and Lower Mantle, edited by C. A. Jones, A. M. Soward and K. Zhang, Taylor and Francis, in press. 
[15] Gubbins D., Barber C. N., Gibbons S. and J. J. Love. Kinematic dynamo action in a sphere. I Effects of differential rotation and meridional circulation on solutions with axial dipole symmetry. Proc. R. Soc. Lond. A, vol. 456 (2000), pp. 1333-1353.

[16] Gubbins D. Can the Earth's magnetic field be sustained by core oscillations. Geophysical Research Letters, vol. 2 (1973), pp. 409-412.

[17] Moffatt H. K. Magnetic Field Generation in Electrically Conducting Fluids Cambridge University Press, (Cambridge, 1978).

[18] Kumar S. and Roberts P. H. A three-dimensional kinematic dynamo. Proc. R. Soc. Lond. A, vol. 344 (1975), pp. 235-258.

[19] Gailitis A., Lielausis O., Platacis E., Dement'ev S., Cifersons A., Gerbeth G., Gundrum T., Stefani F., Christen M. and Will G. Magnetic field saturation in the Riga dynamo experiment. Physical Review Letters, vol. 86 (2001), pp. 3024-3027.

[20] Stieglitz R. And U. Müller Experimental demonstration of a homogeneous two-scale dynamo. Physics of Fluids, vol. 13 (2001), pp. 561-564.

[21] Junk M. And C. EgBers Isothermal spherical Couette flow. In Physics of Rotating Fluids edited by C. Egbers G. Pfister, Springer (Berlin, 2000), pp. 215-233.

[22] Waked A. M. And B. R. Munson. Laminar-turbulent flow in a spherical annulus. Journal of Fluids Engineering, vol. 100 (1978), pp. 281-286.

[23] Frick P., Denisov S., Khripchenko V., Noskov V., Sokoloff D. AND R. StePanov. A nonstationary dynamo experiment in a braked torus. In Dynamo and Dynamics, a Mathematical Challenge, edited by P. Chossat, D. Armbruster and I. Oprea, Kluwer academic publishers, II. Mathematics, Physics and Chemistry - Vol. 26 (Dordrecht, 2001) pp. 1-8.

[24] Duck P. W. And M. R. Foster. Spin-up of homogeneous and stratified fluids. Annual Review of Fluid Mechanics, vol. 33 (2001), pp. 231-263.

[25] Dormy E., Cardin P. And D. Jault. MHD flow in a slightly differentially rotating spherical shell, with conducting inner core, in a dipolar magnetic field. Earth and Planetary Science Letters, vol. 160 (1998), pp. 15-30.

[26] Dormy E., Jault D. And A. M. Soward. A super-rotating shear layer in magnetohydrodynamic spherical Couette flow. Journal of Fluid Mechanics, vol. 452 (2002), pp. 263-291.

[27] Brito D., Cardin P., Nataf H.C. and P. Olson. Experiments on Joule heating and dynamo efficiency. Geophysical Journal International, vol. 127 (1996), pp. 339-347. 
[28] Brito D., Nataf H.-C., Cardin P., Aubert J. and J.-P. Masson. Ultrasonic Doppler velocimetry in liquid gallium. Experiments in Fluids, vol. 31 (2001), pp. 653-663. 

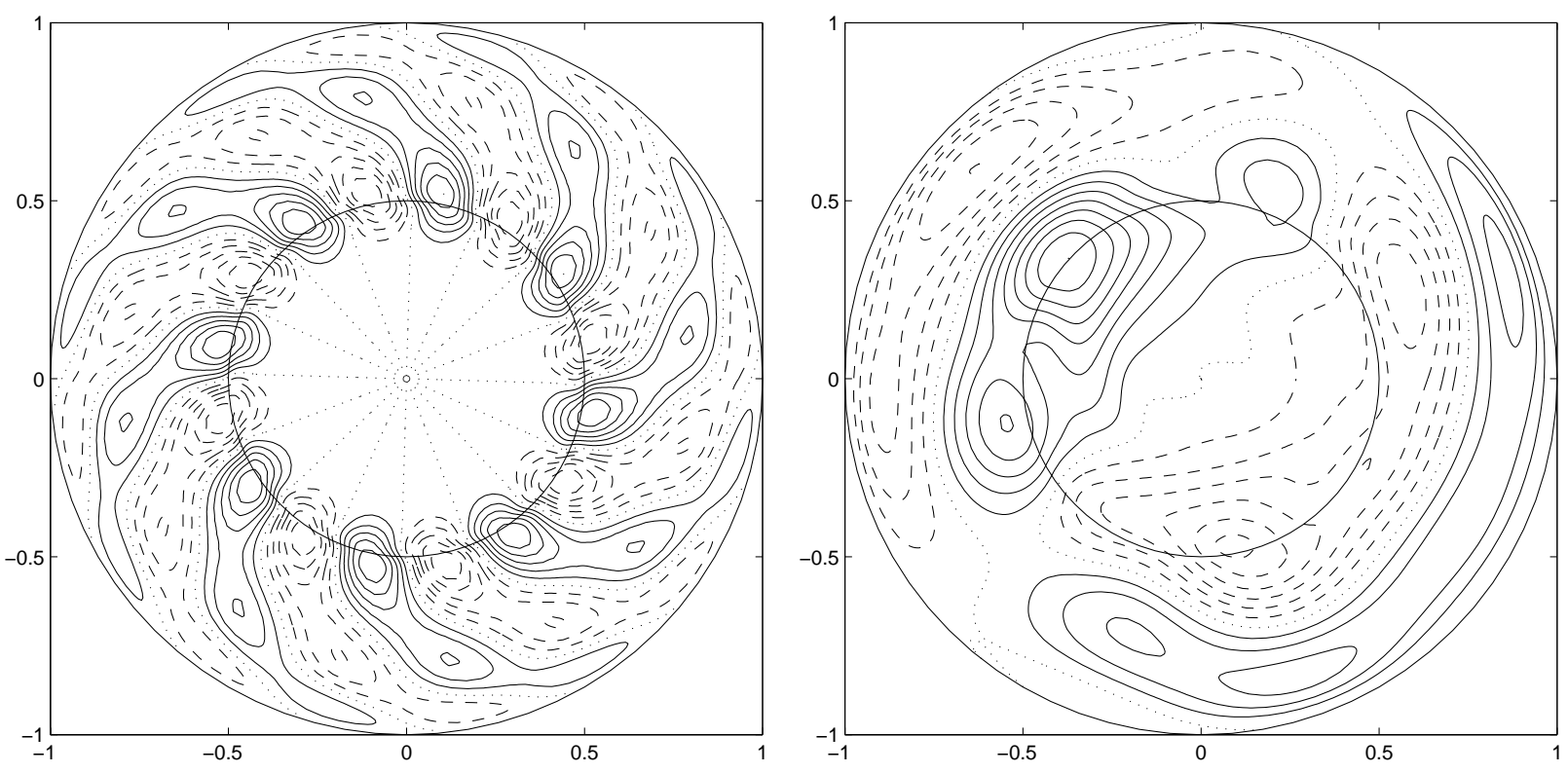

Figure 1: Equatorial cross-section of the flow induced by an unstable viscous shear layer located at a cylindrical radius $s=0.5$ in a rapidly rotating sphere. Two-dimensional numerical simulation assuming Proudman-Taylor invariance along the axis of rotation. Two polar caps $(s<0.5)$ are rotating faster at $\Delta \Omega+\Omega$ than the remaining part of the spherical boundary, which rotates at $\Omega$ $\left(E=10^{-5}\right)$. Lines are contours of the streamfunction of the non axisymmetric flow. On the left, $\Delta \Omega / \Omega=0.033$ gives a typical view of the flow at the onset of instability with a Rossby wave-type flow alike thermal convection. On the right, $\Delta \Omega / \Omega=0.1$ yields a view of the nonlinear regime at 3 times critical. In that case the non-axisymmetric maximum velocity reaches $1 / 3$ of its axisymmetric counterpart. 

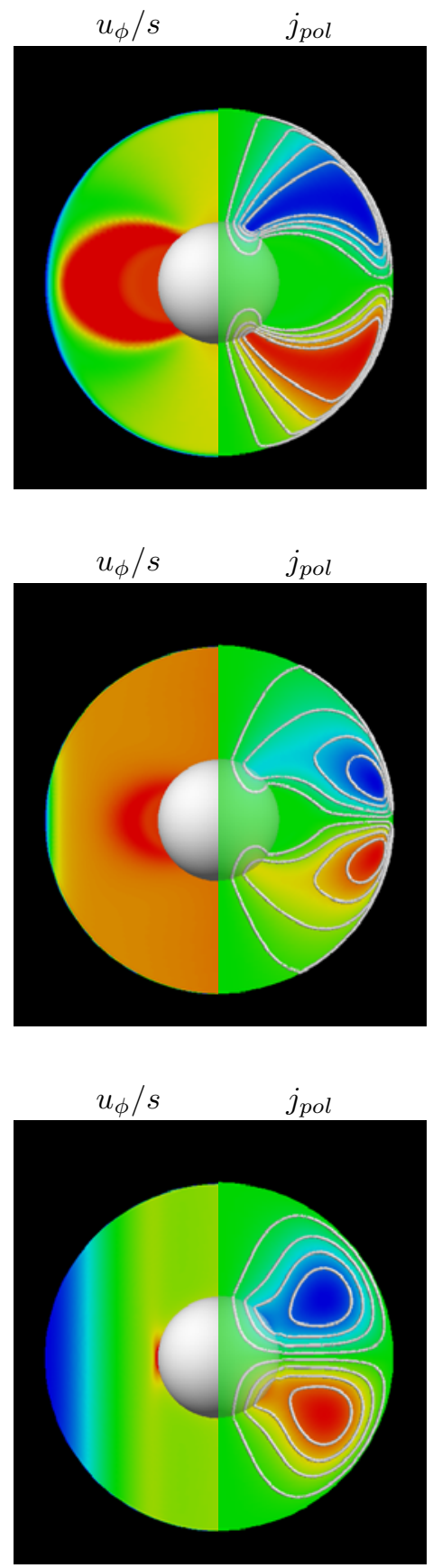

Figure 2: Meridional representations of the zonal angular velocity $u_{\phi} / s$ (left) and meridional electric currents $j_{\text {pol }}$ (right) in a rapidly rotating sphere with a differentially rotating inner sphere permeated by a dipolar magnetic field. From top to bottom, linear simulations that illustrate non rotating, slowly rotating $\left(\Lambda_{i}=1\right)$ and rapidly rotating $\left(\Lambda_{i}=1 \cdot 10^{-2}\right)$ regimes in the DTS experiment. Courtesy of E. Dormy. 
Figure 3: DTS experimental set-up.

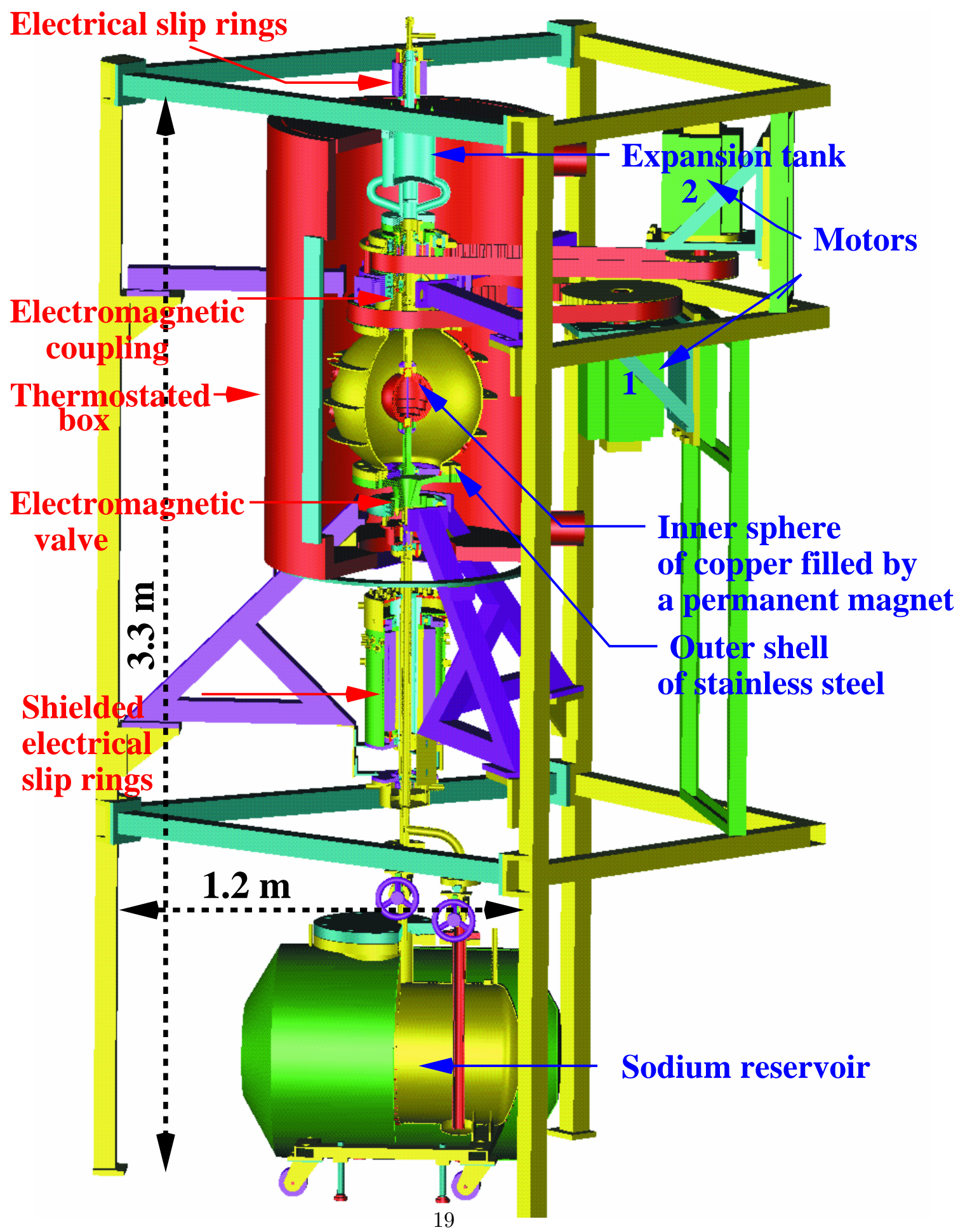

